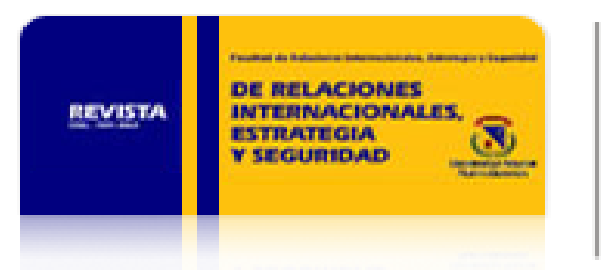

Revista de Relaciones Internacionales, Estrategia y Seguridad

ISSN: 1909-3063

cinuv.relinternal@unimilitar.edu.co

Universidad Militar Nueva Granada

Colombia

Palma, Óscar

GAZA: REFLEXIONES SOBRE LA INTERVENCIÓN HUMANITARIA

Revista de Relaciones Internacionales, Estrategia y Seguridad, vol. 4, núm. 1, enero-junio, 2009, pp.

185-197

Universidad Militar Nueva Granada

Bogotá, Colombia

Disponible en: http://www.redalyc.org/articulo.oa?id=92712970011

- Cómo citar el artículo

- Número completo

- Más información del artículo

- Página de la revista en redalyc.org

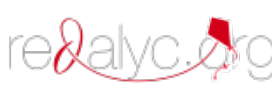

Sistema de Información Científica

Red de Revistas Científicas de América Latina, el Caribe, España y Portugal

Proyecto académico sin fines de lucro, desarrollado bajo la iniciativa de acceso abierto 
rev.relac.int.estrateg.segur.4(1):185-197,2009

\section{GAZA: REFLEXIONES SOBRE LA INTERVENCIÓN HUMANITARIA}

Óscar Palma*

\section{RESUMEN}

El fin del cese el fuego entre Hamas y el Gobierno de Ehud Olmert a finales de 2008 originó una guerra causada por el continuo lanzamiento de cohetes por parte de Hamas al vecino territorio Israelí y la consecuente respuesta de esa nación ocupando la Franja de Gaza. Los fuertes ataques, que repetitivamente afectaron a la población civil y las misiones internacionales, generaron un ambiente de condiciones humanitarias extremas e intolerables en el área más densa del planeta. Esta situación obliga a reflexionar sobre el instrumento de intervención humanitaria.

El mundo entiende dicho instrumento como el medio para intervenir por la fuerza en aquellos Estados donde, por excesos

\footnotetext{
* Internacionalista, Universidad del Rosario. Magíster en Estudios en Seguridad Internacional, Universidad de Leicester. Becario Chevening del Reino Unido. Becario del Centro de Estudios Hemisféricos de Defensa, Universidad Nacional de Defensa, Washington. Investigador línea sobre 'Seguridad Hemisférica', Centro de Estudios Estratégicos en Seguridad y Defensa Nacionales (CEESEDEN). Catedrático universidades Rosario y Militar. omorahh@hotmail.com
} 
del gobierno contra ciertos sectores de la población o grupos subnacionales, se generan emergencias humanitarias supremas. El caso de Gaza demuestra la necesidad contemplar la posibilidad de que dicha intervención no se conciba como exclusiva para escenarios puramente internos, sino también cuando el choque entre uno o más Estados genera las mismas condiciones de degradación humanitaria inaceptable. Sin embargo, se oponen los intereses de las grandes potencias mundiales y sus alianzas, y la posibilidad de que en vez de lograr el control de la situación haya un escalamiento del conflicto.

Palabras clave: Intervención humanitaria, Israel, Gaza, genocidio, Hamas, responsabilidad de proteger, Palestina, guerra de Gaza.

\section{ABSTRACT}

The end of the ceasefire between Hamas and the Government of Ehud Olmert in late 2008 created a war caused by the continued launching of rockets by Hamas onto neighbouring Israeli territory and the consequential response of that nation by occupying the Gaza Strip. The strong attacks which repetitively affected the civilian population and the international missions generated an environment of extreme and intolerable humanitarian conditions in the most densely populated area worldwide. This situation forces a reflection about the instrument of Humanitarian Intervention.

The world understands this instrument as a means to intervene by force in those states where excesses are committed by the government against certain sectors of the population, or sub national groups, generating supreme humanitarian emergencies. What Gaza demonstrates is the necessity to begin thinking about the possibility of conceiving such intervention not as exclusive of purely internal scenarios, but also when the clash between one or more states generates the same conditions of unacceptable humanitarian degradation. However, the interest of world powers and their alliances, together with the possibility of escalating the conflict instead of controlling the situation, opposes such understanding.

Key Words: Humanitarian Intervention, Israel, Gaza, Hamas, genocide, Responsibility to Protect, Palestine, Gaza War.

\section{INTRODUCCIÓN}

La comunidad internacional prometió no volver a permitir los hechos ocurridos el 11 de abril de 1994. Ese día, ante la mirada pasiva de soldados europeos de las Naciones Unidas, cientos de hutus entraron al Ecole Technique Officielle en la ciudad de Kigali, Rwanda, asesinando a 2.500 de sus compatriotas tutsies en el acto (Segal, 2006, p. 48). La Misión de las Naciones Unidas abandonó el sitio ante la imposibilidad de cumplir un papel más determinante en el terreno. Todo ello en 
el marco de un genocidio que cobró la vida de 800.000 personas en tres meses ${ }^{1}$. Es éste otro de los casos que nutren los argumentos existentes sobre la necesidad de que la comunidad internacional cuente con mecanismos de fuerza para permitir intervenciones militares, que ante una catástrofe humanitaria pudieran proveer de un método efectivo para evitar los apocalípticos desenlaces que hasta ahora se han conocido. Casos actuales como el de Darfur, donde las milicias janjaweed persiguen sistemáticamente a la población negra, generarían las condiciones que de plano justificarían dicha intervención.

El caso de la guerra de Gaza (2008-2009), donde nuevamente una comunidad débil y significativamente desprotegida va quedando en condiciones humanitarias intolerables en medio de un enfrentamiento bélico, obliga a hacer una seria reflexión sobre elementos en juego que si bien no son equivalentes a las dinámicas genocidas de Sudan, Rwanda o el holocausto de la Segunda Guerra Mundial, podrían cambiar la concepción que hoy se tiene sobre la intervención humanitaria. Si bien, el caso de Darfur se produce por condiciones internas en medio de la erosión o inexistencia de estructuras de un Estado sólido y, a pesar de que ello puede representar un impacto regional, las dinámicas de Gaza traspasan la orbita exclusiva de un Estado tanto en sus causas como en sus consecuencias.

En esencia, lo que el caso enseñaría es que existen dinámicas que trascienden los límites soberanos de los Estados donde, al igual que en fenómenos internos de persecución sistemática contra grupos subnacionales, se generan situaciones de extrema erosión de los estándares de derechos humanos y donde las condiciones humanitarias en general exigen la acción de la comunidad internacional. La intervención humanitaria debería pensarse también en estos escenarios y no limitarse a ser una herramienta exclusiva para detener atrocidades dignas de regímenes considerados como parias o típicas de Estados fallidos.

En el fondo esta reflexión no sería más que equiparar el sufrimiento de una comunidad humana causada por un choque entre dos o más Estados, con aquella sufrida por los excesos y deseos de un tiránico régimen lanzado al conflicto y la destrucción sistemática. En otras palabras, abrir el debate sobre por qué se viene construyendo una visión que legitima la intervención para el segundo de los casos y no para el primero.

\section{LA INTERVENCIÓN HUMANITARIA}

El concepto de intervención humanitaria viene siendo debatido desde el siglo XVII cuando el tratadista Hugo Grocio consideraba importante rescatar a no-nacionales en peligro, para ser incluidos bajo la esfera de la llamada guerra justa. Pero fue sólo en el Concierto de Europa -siglo XIXcuando comenzó a entrar en práctica (Hough, 2004, p. 117).

${ }^{1}$ The New Republic, The Worst Place, (Vol. 239, No. 4851), diciembre 2008. p.1. 
A pesar de que existe gran variedad de conceptos que buscan delimitar la naturaleza de dicho instrumento, un significado comprehensivo y coherente es presentado por J.L. Holzgrefe quien define a la intervención humanitaria como 'la amenaza o uso de fuerza a través de las fronteras de un Estado, o grupo de Estados, con el objetivo de prevenir o dar fin a la extendida y grave violación de los derechos humanos fundamentales de los individuos diferentes a sus ciudadanos, sin el permiso del Estado donde se aplica la fuerza (Holzgrefe, 2003, p. 18).

El planteamiento de un instrumento de esta naturaleza genera una serie de dilemas de naturaleza política y jurídica que no dejan de ser complejos. Políticamente, la intervención representa un desafío al concepto de soberanía que ha sido la columna vertebral del orden Westfaliano. Desde la aparición de los Estados-Nación viene haciendo carrera la creencia de que la capacidad y facultad de estos actores de regirse a sí mismos y controlar las dinámicas al interior de sus fronteras no puede ser irrumpida por algún otro actor del sistema internacional, mucho menos por medio de la fuerza. Teniendo en cuenta que en la mayoría de circunstancias los fenómenos que justifican una intervención se presentan en la orbita interna de los Estados, la fórmula resulta ser la autorización del uso de fuerza en espacios donde la soberanía de un Estado es tradicionalmente inviolable.

Transformar el entendimiento de soberanía que ha regido desde el siglo XVII para darle legitimidad a la intervención puede conllevar un gran riesgo. ¿Cómo garantizar que un Estado -o los estadosno utilicen este instrumento para justificar alguna intervención en persecución de algún interés que supera la dimensión puramente humanitaria? No debe olvidarse que uno de los argumentos utilizados para derrocar a Saddam Hussein fue la múltiple violación de derechos de las comunidades iraquíes ${ }^{2}$, especialmente la masacre de Halabja, donde fallecieron de 3.500 a 5.000 kurdos a causa de una serie de ataques utilizando arsenales químicos (Kasey, 2006, p. 61).

Aquí cabe una pregunta, ¿obedeció la guerra de Irak a una motivación puramente humanitaria por parte de Washington y los Aliados, tal como debería darse en una intervención humanitaria? Seguramente en la balanza de motivaciones para la Casa Blanca, la posibilidad de iniciar una presencia estratégica en el Medio Oriente, la cuestión de los recursos energéticos y hasta la preocupación por la existencia de arsenales de destrucción masiva pesaron mucho más que el maltrato a la población iraquí. Precisamente, esta invasión ha sembrado dudas en la comunidad internacional para avanzar en el tema de la intervención humanitaria. Se teme que el escenario de guerra civil que se experimenta tras la caída de Hussein se replique en otros escenarios, o que se utilice la bandera de la justicia humanitaria en teatros de guerra que persiguen otros intereses.

La cuestión de la soberanía viene siendo solucionada desde principios de la presente década. EI dilema motivó a las Naciones Unidas a convocar la Comisión Internacional en Intervención y soberanía del Estado -ICISS- en 2001, buscando lograr un equilibrio entre los conceptos de inter-

${ }^{2}$ US Department of State «Life Under Saddam Hussein: Past Repression and Atrocities by Saddam Hussein's Regime» Apr. 4, 2003, http://www.state.gov/p/nea/rls/19675.htm, Consultado March 30, 2007

GAZA: REFLEXIONES SOBRE LA INTERVENCIÓN HUMANITARIA 
vención y soberanía. El resultado fue un nuevo marco conceptual de análisis y acción conocido como la Responsabilidad de Proteger (Mac Farlane, Thielking y Weiss, 2007, p. 978). La concepción cambió de soberanía como control a soberanía como responsabilidad. Esto es, el Estado tiene la obligación de garantizar la seguridad y derechos a sus ciudadanos, no a ejercer un control de facto. Si no está en esta capacidad, su soberanía carecería de sentido y sería la comunidad internacional quien debería garantizar los derechos de esa nación (o grupo subnacional). En el fondo, esto no es más que la aplicación de lo que Robert Keohane denomina gradaciones de la soberanía. Es decir, el entendimiento no de un concepto monolítico, sino la existencia de diversos niveles o tipos de soberanía (Keohane, 2007, p. 277). Por tanto, la acción de fuerza resulta coherente y legítima³

Actualmente en el marco del debate sobre intervención humanitaria se discute si dicho instrumento debe ser esencialmente militar o si debe ampliarse para incluir herramientas de naturaleza política, social y económica. Esto, sin duda, crearía una especie de área gris entre lo que es la intervención humanitaria pura y las dinámicas de peacekeeping y peacebuilding. Pero hasta ahora no se trata, por lo menos a nivel conceptual, la posibilidad de una intervención humanitaria en un conflicto resultante del enfrentamiento entre dos Estados.

Más complejo aún resulta el panorama de la legalidad, pues las normas de Derecho Internacional parecerían oponerse a la idea misma de intervención. Dos máximas del Derecho son la abstención del uso de fuerza y la no intervención en los asuntos internos de los Estados, materializados en la Carta de las Naciones Unidas. El Artículo 2.4 afirma:

«Los Miembros de la Organización, en sus relaciones internacionales, se abstendrán de recurrir a la amenaza o al uso de la fuerza contra la integridad territorial o la independencia política de cualquier Estado, o en cualquier otra forma incompatible con los Propósitos de las Naciones Unidas».

Por su parte, el Artículo 2.7 afirma:

«Ninguna disposición de esta Carta autorizará a las Naciones Unidas a intervenir en los asuntos que son esencialmente de la jurisdicción interna de los Estados, ni obligará a los Miembros a someter dichos asuntos a procedimientos de arreglo conforme a la presente Carta; pero este principio no se opone a la aplicación de las medidas coercitivas prescritas en el Capítulo VIl».

La última determinación de este artículo, sin embargo, abre la posibilidad a una interpretación diferente de las normas afirmando que a pesar de que la intervención humanitaria no se expresa textualmente en la Carta de las Naciones Unidas, está implícita en diversas respuestas de la comunidad internacional a las agresiones a la paz, ya que se autorizan medidas coercitivas a través del

\footnotetext{
${ }^{3}$ Para profundizar en el debate sobre la intervención humanitaria y la responsabilidad de proteger, leer T. Farer, D. Archibugi, C. Brown, N.C, Crawford, T.G. Weiss y N. J Wheeler, «Roundtable: Humanitarian Intervention After 9/11» in International Relations 2005, Vol. 19, No. 2, pp. 211-250.
} 
Capítulo VII. Este capítulo -Acciones en caso de amenazas a la paz, Quebrantamientos de la Paz o Actos de Agresión- autoriza el uso de fuerza en cabeza del Consejo de Seguridad si se llegasen a producir los eventos a los que se hace referencia en su título. Es bajo esta disposición como se hace posible la intervención humanitaria, pues el Consejo de Seguridad podría determinar las acciones militares correspondientes sin que exista una transgresión al Derecho Internacional. Se responde así al primero de los principios de las Naciones Unidas consagrado en el Artículo 1.1:

«Mantener la paz y la seguridad internacionales, y con tal fin: tomar medidas colectivas eficaces para prevenir y eliminar amenazas a la paz, y para suprimir actos de agresión u otros quebrantamientos de la paz; y lograr por medios pacíficos, y de conformidad con los principios de la justicia y del derecho internacional, el ajuste o arreglo de controversias o situaciones internacionales susceptibles de conducir a quebrantamientos de la paz».

De esta forma se configura un ejercicio casuístico de intervenciones humanitarias, que termina generando una costumbre sobre las acciones de la comunidad cuando aparece una emergencia humanitaria suprema que requiere de la acción internacional. Y es aquí donde surge el debate sobre la materia legal. Por un lado, una visión positivista del Derecho que exigiría la codificación precisa del procedimiento y las motivaciones que deben existir para una intervención legal y legítima. Ello demandaría una reforma a la Carta de las Naciones Unidas y un consecuente proceso extenso y complejo. Por otro lado, la posición 'costumbrista' que aboga por mantener la posibilidad de tomar decisiones en la dinámica de caso por caso.

En medio de este panorama, ¿qué criterios pueden determinar la justificación de una intervención humanitaria legítima? La Responsabilidad de Proteger (R2P) cita dos requisitos: intención correcta y autoridad correcta. El primero indica que la acción militar debe estar dirigida únicamente a detener o evitar el sistemático sufrimiento humano. El segundo ocurre cuando, bajo el Derecho Internacional existente, el Consejo de Seguridad de la ONU autoriza el procedimiento. En su defecto, la Asamblea General o una Organización Regional bajo disposiciones del capítulo VII de la carta de la ONU.

Los criterios delineados por la ICISS evitarían que en la búsqueda de intereses particulares de un Estado se clame una emergencia humanitaria como excusa para actuar militarmente.

Por otro lado, y desde el pensamiento académico, Tom Farer, quien además de haber sido Presidente de la Comisión Interamericana de Derechos Humanos de la OEA ha ocupado numerosas posiciones en diversas Universidades del mundo, establece unos parámetros útiles para determinar cuándo debe ejecutarse una intervención humanitaria (T. Farer, D. Archibugi, C. Brown, N.C. Crawford, T.G. Weiss y N. J Wheeler, 2005, p. 215):

1. Debe existir una emergencia humanitaria suprema.

2. Todas las formas no violentas de solución deben haberse agotado y deben haber sido infructuosas.

GAZA: REFLEXIONES SOBRE LA INTERVENCIÓN HUMANITARIA 
3. La intervención debe ser conducida de acuerdo al Derecho Internacional y el daño debe ser menor comparado con el sufrido por la población si la intervención no ocurriera.

4. Debe haber una alta probabilidad de que el uso de la fuerza va a llevar a un resultado positivo.

5. El interventor debe reportar la intervención al Consejo de Seguridad y solicitar que sus acciones sean monitoreadas."

Estos criterios serán útiles para analizar el caso particular de Gaza.

\section{LAS REALIDADES EN GAZA}

Existen dos sectores políticos en Palestina. Por un lado, el Fatah, obra de Yasser Arafat, hoy en manos del Presidente Mahmoud Abbas (Abu Mazen) con una posición relativamente conciliadora y abierta a la negociación con los israelitas. Por otro, Hamas, partido político considerado como organización terrorista por Estados Unidos y Europa ${ }^{4}$, de corte radical, no sólo cerrado a las negociaciones con el pueblo judío, como lo explica el experto en asuntos religiosos de Medio Oriente Litvak Meir, sino promotor de su eliminación como comunidad política organizada en el Medio Oriente (Meir, 2005, pp. 41-43).

Dos factores catapultaron el protagonismo de Hamas en el escenario político. Por un lado, la realización de elecciones en el 2005 donde Hamas superó a Fatah por 44,45\% contra 44,41\% (Ferre, 2006, p. 2). Por otro, la salida unilateral de Israel de la franja de Gaza que permitió a Hamas ocupar este espacio por la fuerza. Se dividió así Palestina con el gobierno de Fatah en la ribera occidental y el de Hamas en Gaza.

Como medida de presión, Israel cerró los cruces fronterizos generando un bloqueo económico de delicadas consecuencias. Hamas inició una campaña de ataque con cohetes Qassam impactando territorio israelí cercano a la franja. La situación humanitaria se tornó complicada, con un gobierno sin recursos, con una notable dificultad para conseguir alimentos y proveer de energía y servicios a la población. Se firmó un cese al fuego entre Hamas e Israel en junio de 2008 que cayó en diciembre, momento en el cual se intensifica el disparo de cohetes $^{5}$.

La respuesta de Israel fue desproporcionada, no sólo haciendo referencia al alto porcentaje de civiles asesinados como se ilustra más adelante, sino causando también la destrucción de propiedades civiles. Se atacaron mezquitas y una universidad bajo el argumento de que allí funcionaban centros de apoyo o armerillos de Hamas. Se asesinaron miembros de misiones médicas y de

\footnotetext{
${ }^{4}$ Department of State, Foreign Terrorist Organizations, 2005. Online en http://www.state.gov/s/ct/rls/fs/
} 37191.htm 2005. Consultado en enero 10, 2009.

${ }^{5}$ AlJazeera, Timeline: Gaza Crisis, Enero 21, 2009. Online en: http://english.aljazeera.net/news/middleeast/ 2009/01/200917205418665491.html. Consultado en enero 21, 2009. 
asistencia de las Naciones Unidas y se atacaron tres instalaciones de la $\mathrm{ONU}^{6}$, una de ellas con fósforo blanco, elemento que generó polémica por su posible utilización extendida en toda Gaza ${ }^{7}$.

La población de la franja cayó en una delicada situación humanitaria. Al inicio de los ceses unilaterales del fuego, 1.326 personas habían muerto, incluyendo 417 menores de edad y por lo menos 660 civiles. 5.400 heridos se sumaban a la cuenta ${ }^{8}$. Del lado Israelí, cayeron 13 personas, entre ellos tres civiles y cuatro bajas que se dieron entre propias tropas ${ }^{9} .4 .000$ edificios fueron destruidos y más de 20.000 quedaron en delicadas condiciones estructurales. 50.800 personas habían sido despojadas de sus hogares y 400.000 sin posibilidades de abastecerse de agua ${ }^{10}$.

Las cifras podrían quedar cortas para explicar la precaria realidad humanitaria en Gaza. Los ataques generaron una serie de condiciones estructurales que dejaron en extremo riesgo al millón y medio de habitantes de la región. La intensidad de los bombardeos en la zona con la mayor densidad de población mundial $\left(4.168 \mathrm{hab} / \mathrm{km}^{2}\right)^{11}$ hizo que cada ataque tuviera un efecto colateral considerable, gestándose un ambiente de inseguridad, angustia e incertidumbre permanentes. Esto, teniendo en cuenta el nivel de destrucción de residencias civiles, hizo que el desplazamiento fuera elevado. La escasez de alimentos, que si bien era significativa antes de los ataques por el bloqueo económico -más del $80 \%$ de la franja dependía de la ayuda humanitaria de la ONU ${ }^{12}$ - naturalmente se incrementó y mucho más si se cuentan las dificultades que las operaciones le impusieron al desarrollo de actividades de la Agencia de las Naciones Unidas para la Protección de los Refugiados en Palestina-UNRWA-, no sólo por la dificultad de transitar el territorio, sino por un ataque que destruyó una considerable cantidad de alimentos.

\footnotetext{
${ }^{6}$ BBC News, Gaza Crisis: Key Maps and Timeline, Enero 14, 2009. Online en: http://news.bbc.co.uk/2/hi/ middle_east/7812290.stm. Consultado en: Enero 15, 2009.

${ }^{7}$ El fósforo blanco es un sólido grasoso que se quema fácilmente y es utilizado en manufacturas químicas y bombas de humo. La exposición a este elemento puede causar quemaduras, daños de hígado, riñón, corazón, pulmón; o la muerte. [Traducción del autor]. Agency for Toxic Substances and Disease Registry. ToxFAQs for White Phosporus, Septiembre 1997. Online en http://www.atsdr.cdc.gov/tfacts103.html. Consultado en febrero 27, 2009.

${ }^{8}$ Oficina Central de Estadísticas de la Autoridad Palestina, Boletín Electrónico Mensual, 15 de diciembre - 15 de enero. No. 30. enero 15, 2009. Online en: http://www.pcbs.gov.ps/Portals/PCBS/Documents/ Monthly\%20bulletin_30/Monthly\%20bulletin_e.htm. Consultado en enero 21, 2009.

${ }^{9}$ The Guardian, Israeli Human Rights Group Speak Out as Death Toll Passes 1000. Enero 15, 2009. Online en: http://www.guardian.co.uk/world/2009/jan/15/human-rights-gaza-israel. Consultado en enero 20, 2009.

${ }^{10}$ BBC News, Gaza Looks like Earthquake Zone. Enero 20, 2009. Online en: http://news.bbc.co.uk/2/low/ middle_east/7838618.stm, Consultado en enero 21, 2009.

${ }^{11} \mathrm{CIA}$ World Factbook, Gaza Strip, 2008. Online en: https://www.cia.gov/library/publications/the-worldfactbook/geos/gz.html. Consultado en Enero 21, 2009.

${ }^{12}$ BBC News, Fuel Crisis halts Gaza Fuel Aid, abril 24, 2008. Online en http://news.bbc.co.uk/2/hi/middle_east/ 7364172.stm. Consultado en enero 21, 2009

GAZA: REFLEXIONES SOBRE LA INTERVENCIÓN HUMANITARIA
} 
Por otro lado, el combustible fue siempre escaso desde que Israel implementó el bloqueo (Ómer, 2008, p. 18), mientras la prestación de servicios públicos se dificultó enormemente. El sistema de salud colapsó, según el Journal británico de salud The Lancet, ${ }^{13}$ mientras las condiciones sanitarias eran paupérrimas. A mediano plazo y si hubieran continuado las acciones de destrucción en un medio urbano tan concentrado, podría haberse presentado un panorama muy similar a las denigrantes condiciones de enfermedad, morbilidad y contaminación vividas durante el rattenkrieg de Stalingrado en la Segunda Guerra Mundial.

\section{3. ¿INTERVENCIÓN EN GAZA?}

A la luz del tema de intervención humanitaria, cabría hacerse varios cuestionamientos: ¿es comparable la situación producida en Gaza tras las acciones militares de Israel con anteriores crisis humanitarias?, ¿ puede justificarse una intervención en esa región?

Esta reflexión no resulta ser un ejercicio puramente coyuntural, pues independientemente de si el cese el fuego es perdurable, las condiciones de una emergencia humanitaria existieron y dado el carácter internacional presente, la reflexión puede ser útil para el entendimiento de la intervención humanitaria a futuro en un contexto más amplio.

Comparar la situación en Gaza con otras catástrofes humanitarias o genocidios históricos puede no llegar a ser un ejercicio fructífero por las dimensiones y motivos existentes. Hitler arrasó con seis millones de judíos, Turquía con 1,6 millones de armenios (Hough, 2004, p. 108) y los hutus con 800.000 tutsies. Si bien, las cifras en Gaza son infinitamente menores, es necesario reflexionar sobre la proporcionalidad, pues los espacios geográficos y los volúmenes de las poblaciones son incomparables. Por otro lado, si bien en todos los casos se observa una conducta sistemática de aniquilamiento intencionado, en el caso de Gaza, Olmert no buscaba arrasar con los palestinos. De tal forma, afirmar que allí se cometió un genocidio puede llegar a ser erróneo e impreciso.

Surge aquí una primera reflexión. ¿Es acaso la intervención humanitaria un instrumento exclusivo frente al genocidio? La respuesta no debe ser afirmativa. Por un lado, el hecho de que no exista una intención de aniquilar a la población de Gaza no significa que las realidades estructurales resultantes del conflicto no hayan creado un ambiente de permanente y grave emergencia humanitaria que bien justificaría una inmediata reacción internacional.

Si se cumple la justa causa esbozada por la R2P, una intervención humanitaria resultaría legítima y es evidente que en Gaza existió una justa causa. Igualmente, siguiendo los criterios sugeridos por

\footnotetext{
${ }^{13}$ Le Monde, La revue medicale The Lancet accuse a Israel d'attrocittes, Enero 15, 2009. Online en : http:// www.lemonde.fr/proche-orient/article/2009/01/15/la-revue-medicale-the-lancet-accuse-israel-datrocites_1142039_3218.html\#ens_id=1106055. Consultado en enero 15, 2009.
} 
Farer, la emergencia humanitaria suprema existiría, legitimando la acción. Por supuesto la cuestión no es sencilla y es necesario detenerse en otro par de elementos enmarcados en los requerimientos de Farer.

Los recursos de negociación y de solución por vías pacíficas previos al cese al fuego no habían sido agotados, de tal forma una intervención habría resultado anacrónica y probablemente contraproducente. En Cairo y Sharm-el-Sheik se fueron presentando intensos esfuerzos diplomáticos para encontrar una solución ${ }^{14}$. Si la violencia se hubiese reestablecido, y de fracasar todo intento de solución diplomática, ¿se habría dejado a la población de Gaza a merced de las armas?

Es claro que más difícil aun resultaría cumplir el requisito de que la violencia generada por la intervención debe ser menor a aquella experimentada de continuarse las hostilidades, y ello se debe a varios factores.

La intervención humanitaria se piensa en un escenario donde la violencia inicial obedece a dinámicas internas. Es el caso de Rwanda, Serbia, Irak y Darfur. A pesar de que Palestina no tiene un territorio definido, podría decirse que las acciones entre judíos y palestinos desbordan la órbita de lo puramente interno. Y cuando existen actores internacionales inmersos en una dinámica de conflicto, la imparcialidad requerida para determinar objetivamente la necesidad de una intervención por motivos humanitarios languidece ante la fortaleza de las alianzas y los intereses de cada Estado.

La intervención podría tener tres ópticas. Entrar al teatro como un ente neutral que separa las líneas enemigas mientras un cese el fuego o el fin de hostilidades son negociados. Este modelo tiene un riesgo y es que podría simplemente aplazar la violencia que ineludiblemente se reanudará más adelante, cayendo en el escenario de la Ecole Technique Ofíciele de Kigali.

Por el contrario, se podría entrar al escenario como un ente neutral pero enfrentando con fuerza a los dos bandos, obligándolos a capitular. Ello muy seguramente redundaría en un recrudecimiento de las hostilidades y el escalamiento del conflicto. Autores como Alan Kuperman ponen en duda la intervención humanitaria precisamente porque la violencia tiende a expandirse más rápido que la posibilidad de los interventores de actuar; y porque, en general, la intervención requiere ponerse del lado de un grupo subnacional legitimándolo ante su enemigo (Kuperman, 2004, p. 65-66).

Como última opción, está la de intervenir atacando a una de las partes. Este modelo tiende a ser exitoso, como lo fue en los Balcanes, y seguro ayudaría a la crisis de Darfur, pero para el caso de Gaza tendría que atacarse a Israel y ahí surgen dos masivos dilemas.

El primero es la capacidad militar de ese país. Las intervenciones se han hecho contra actores débiles o que no poseen una capacidad que pudiera poner en riesgo a las potencias militares y en

${ }^{14}$ France 24, Ceasefire negotiations stepped up in Cairo. Enero 14, 2009. Online en: http://www.france24.com/ en/20090114-gaza-ceasefire-negotiations-stepped-up-cairo-. Consultado en enero 21, 2009.

GAZA: REFLEXIONES SOBRE LA INTERVENCIÓN HUMANITARIA 
últimas a la estabilidad internacional. Israel es un país que cuenta no sólo con excepcionales fuerzas convencionales, sino posiblemente con capacidades nucleares. Ineludiblemente la intervención, en este caso, amenazaría con transformarse en una guerra internacional de considerables condiciones.

Segundo, y ejemplificando lo que se enunciaba anteriormente, Israel es un aliado occidental y ello implica que muy seguramente no habría consenso para la intervención. Es aquí cuando verdaderamente se aprecia el relativismo subjetivo de la intervención humanitaria. Se usa contra enemigos con quienes no existe vínculo de interés, no por la intención pura de responder a una crisis humanitaria. ¿Qué pasaría si fuera Irán y no Israel quien estuviera cometiendo estas acciones? ¿Existiría, ahí sí, un gran concierto para atacar? Es aquí donde deben comenzar a generarse los cambios en aras de rescatar un verdadero instrumento de intervención humanitaria.

Se estaría, eso sí, ante una línea divisoria muy gris entre el ámbito de la guerra internacional y el ámbito puro de la intervención. Se podría argumentar que en toda guerra existe una erosión de las condiciones humanas como consecuencia natural de las operaciones militares, y que en ese sentido la participación de un poder externo a la dinámica no terminaría siendo una intervención humanitaria per se, sino una simple participación en la guerra. Dos elementos pueden ser diferenciadores.

Primero, en el análisis de la justa causa, debe pesar la realidad de la asimetría estratégica entre actores en un conflicto donde una comunidad específica resulta ser la principal víctima de las hostilidades, lo que se manifiesta en las denigrantes realidades humanitarias del escenario. No en toda guerra internacional la principal víctima resulta ser una comunidad civil cuyas condiciones de vida se minimizan a niveles inaceptables como en Gaza o Stalingrado.

Segundo, la intervención no puede entenderse como realizada por un solo Estado o una alianza de Estados, pues eso sería precisamente una guerra internacional. Debe ser una expresión legal y legítima de la comunidad internacional, de acuerdo al Derecho Internacional. De ahí la importancia que Farer reconoce tanto de este tema, como de la necesidad de reportar la actividad al Consejo de Seguridad. Evidentemente, la comunidad internacional esta aun lejos de lograr este nivel de consenso, pero es necesario, por lo menos, comenzar a considerarlo.

\section{CONCLUSIÓN}

El caso de Gaza genera una dicotomía. Si bien, epitomiza la posibilidad de pensar la intervención humanitaria más allá de la tradicional óptica en contra de regímenes parias que abusan de sus mismas comunidades, el análisis de las consecuencias de la generación de violencia producida por la intervención iría en contravía de los principios de acción de la misma. El resultado, por tanto, sería catastrófico. Pero esto no implica que, cuando se trata de un escenario con elementos internacionales, el resultado siempre sea una catástrofe mayor, abriendo la posibilidad de por lo menos considerar éstos como escenarios válidos para la intervención humanitaria. 
Existe gran duda de que la comunidad internacional logre acordar una posición definitiva respecto a la intervención humanitaria en escenarios con elementos internacionales. Difícil es separar las intenciones puramente humanitarias de los intereses específicos de cada nación y su materialización a través de alianzas estratégicas. Una posible vía, a muy largo plazo, sería lograr un consenso sobre el proceder en la intervención bajo la percepción definida hoy y luego evaluar la posibilidad de aplicar el proceso en dinámicas que involucran a dos o más Estados.

\section{BIBLIOGRAFÍA}

\section{Fuentes secundarias}

Ferre, J.M. 2006. «Consecuencias de la victoria de Hamas en las elecciones palestinas». En Instituto Real Elcano de Estudios Internacionales y Estratégicos, ARI, no. 18, febrero 20, p. 2. Online en: http:// www.realinstitutoelcano.org/analisis/911/911_Ferre.pdf. Consultado en enero 21, 2009.

Farer, T., Archibugi, D., Brown, C., Crawford, N.C., Weiss, T.G. y Wheeler, N. J. 2005. «Roundtable: Humanitarian Intervention After 9/11». En International Relations, vol. 19, no. 2.

Hough, P. 2004. Understanding Global Security. London, Routledge.

Kasey, L. 2006. «Questioning Halabja». En Dissent, verano, vol. 50, no. 3.

Keohane, R. y Holzgrefe, J.L. 2003. Humanitarian Intervention: Ethical, Legal and Political Dilemmas. Cambridge: Cambridge University Press.

Kuperman, A. 2004. «Humanitarian Hazard». En Harvard International Review, primavera, vol. 26, no. 1.

Mac Farlane, S.N., Thielking, C. y Weiss, T.G. 2004. «The Responsibility to Protect: Is Anyone Interested in Humanitarian Intervention?». En Thirld World Quarterly, vol. 25, no. 5.

Meir, L. 2005. «The Anti-semitism of Hamas». En Palestine-Israel Journal, vol. 12, no. 2/3.

Omer, M. 2008. «Dead in the Water: Israel's Fuel Blockade on Gaza». En Washington Report on Middle East Affairs, julio, vol. 27, no. 5.

Seagal, V. 2006. «A Certain Death». En New Statesman, abril 6, vol. 135, no. 4786.

The New Republic. 2008. The Worst Place, vol. 239, no. 4851. 


\section{Fuentes institucionales}

CIA World Factbook. 2008. Gaza Strip. Online en: https://www.cia.gov/library/publications/the-worldfactbook/geos/gz.html. Consultado en enero 21, 2009.

Department of State. 2005. Foreign Terrorist Organizations. Online en http://www.state.gov/s/ct/rls/fs/37191.htm 2005. Consultado en enero 10, 2009.

Oficina Central de Estadísticas de la Autoridad Palestina. 2009. Boletín Electrónico Mensual, 15 de diciembre - 15 de enero. No. 30, enero 15. Online en: http://www.pcbs.gov.ps/Portals/PCBS/Documents/ Monthly\%20bulletin_30/Monthly\%20bulletin_e.htm. Consultado en enero 21, 2009.

United Nations. 2005. Charter of the United Nations and Statute of the International Court of Justice. New York: UN Department of Public Information.

US Department of State. 2003. «Life Under Saddam Hussein: Past Repression and Atrocities by Saddam Hussein's Regime» Apr. 4. http://www.state.gov/p/nea/rls/19675.htm. Consultado en marzo 30, 2007.

\section{Fuentes noticiosas}

AlJazeera, Timeline: Gaza Crisis, Enero 21, 2009. Online en: http://english.aljazeera.net/news/middleeast/ 2009/01/200917205418665491.html. Consultado en enero 21, 2009.

BBC News, Gaza Crisis: Key Maps and Timeline, Enero 14, 2009. Online en: http://news.bbc.co.uk/2/hi/ middle_east/7812290.stm. Consultado en enero 15, 2009.

BBC News, Gaza Looks like Earthquake Zone. Enero 20, 2009. Online en: http://news.bbc.co.uk/2/low/ middle_east/7838618.stm, Consultado en enero 21, 2009.

BBC News, Fuel Crisis halts Gaza Fuel Aid, abril 24, 2008. Online en http://news.bbc.co.uk/2/hi/middle_east/ 7364172.stm. Consultado en enero 21, 2009.

France 24, Ceasefire negotiations stepped up in Cairo. Enero 14, 2009. Online en: http://www.france24.com/ en/20090114-gaza-ceasefire-negotiations-stepped-up-cairo-. Consultado en enero 21, 2009.

Le Monde, La revue medicale The Lancet accuse a Israel d'attrocittes, Enero 15, 2009. Online en: http:// www.lemonde.fr/proche-orient/article/2009/01/15/la-revue-medicale-the-lancet-accuse-israel-datrocites_1142039_3218.html\#ens_id=1106055. Consultado en enero 15, 2009.

The Guardian, Israeli Human Rights Group Speak Out as Death Toll Passes 1000. Enero 15, 2009. Online en: http://www.guardian.co.uk/world/2009/jan/15/human-rights-gaza-israel. Consultado en enero 20, 2009.

The Times, UN Headquarters in Gaza Hit by White Phosphorus Mortar Shells, Enero 15, 2009. Online en: http://www.timesonline.co.uk/tol/news/world/middle_east/article5521925.ece. Consultado en enero 15, 2009. 